\title{
Studies of infective endocarditis in cardiomyoblasts H9c2 cell line
}

\begin{abstract}
Infective endocarditis (IE) is a condition that could possibly be induced by poor oral hygiene. Peptidoglycan (PGN) and lipoteichoic acid (LTA) promote cytokine expression and nitric oxide (NO) production by binding toll-like receptor (TLR)-2, considered the innate immune response initiator. Some studies have indicated that flavonoids possess antioxidant and anti-inflammatory properties. The aim of this review consisted in describe in vitro studies related to PGN and LTA in IE in H9c2 cell line.
\end{abstract}

Keywords: infective endocarditis, anti-inflammatory properties, flavonoids possess, valve lesions, hemodialysis, heteropolymer, gram positive bacteria, microorganisms, glucosamine, osmolarity, external turgescence
Volume 4 Issue $6-2017$

\section{Berenice Fernández Rojas, Juan Arturo \\ Gómez Mora, Marisol Rosas Martínez, Gutiérrez Venegas Gloria}

Faculty of Dentistry, National Autonomous University of México, México

\author{
Correspondence: Gutiérrez-Venegas Gloria, Faculty of \\ Dentistry, National Autónomas University of México and \\ Laboratory of Biochemistry of the División of Postgraduate \\ Studies and Research, Faculty of Dentistry, Ciudad Universitaria, \\ 04510 México, Email Mexicogloria@fo.odonto.unam.mx
}

Received: June 01, 2017 | Published: June 23, 2017

Abbreviations: IE, infective endocarditis; PGN, peptidoglycan; LTA, lipoteichoic acid; NO, nitric oxide; TLR, toll-like receptor; PAMP, pathogen-associated molecular patterns; TNF, tumor necrosis factor; ROS, reactive oxygen species; PG, plus peptidoglycan

\section{Review}

Infective endocarditis (IE) is the inflammatory process that triggers infections in different heart locations such as heart valves, septal defects, tendon cords and the endocardium. As main risks factors for IE disease is the presence of chronic vascular accesses and immune system diseases, out of which the bicuspid valve might be the most affected. Although factors such as age, gender and use of intravenous drugs are also relevant, other risks factors include chronic rheumatic heart disease (which now accounts for $<10 \%$ of cases in industrialized countries), age-related degenerative valve lesions, hemodialysis, and coexisting conditions such as diabetes, human immunodeficiency virus infection, and intravenous drug use. Antibiotic therapy is the most employed means to treat IE. In developed countries found ratio was 3 to 10 cases out of every 100000 subjects, females were more affected than males.IE is associated with cultures of Gram positive bacteria, including Staphylococcus aureus, Streptococcus bovis and Streptococcus viridians, as well as cultures with Gram negative bacteria, such as Haemophilus, Aggregatibacter, Cardio bacterium, Eikenella and Kingella, out of which, Streptococcus and Staphylococcus are microorganisms most frequently associated in several types of IE; all are present in dental biofilm. ${ }^{1}$

Bacteria could be disseminated into the bloodstream by means of poor oral hygiene, tooth extraction procedures and periodontal treatments. ${ }^{2}$ Immune system is activated after recognizing pathogenassociated molecular patterns (PAMPs) present in the cell wall of microorganisms such as LTA and PGN, also known as murein, which is a heteropolymer formed by an alternating sequence of $\mathrm{N}$-acetyl glucosamine and $\mathrm{N}$-acetylmuramic acid crosslinked with a short peptide ( $\beta-1,4$ bonds). ${ }^{3}$ PGN forms an elastic mesh that covers the entire surface of the bacteria; it plays a protective role from cellular damage, allowing it to withstand the pressure of external turgescence due to high osmolarity of the cytoplasm conferring bacteria shape. ${ }^{4}$ This component is found in both Gram positive and negative bacteria. On the other hand, LTA is the major component of the cell wall of Gram positive bacteria. ${ }^{5,6}$ It is composed of a backbone of repeating glycerophosphate units with D-alanine or N-acetyl glucosamine substituent and a lipophilic anchor. ${ }^{7}$ Recently it has been suggested that LTA possesses inflammatory properties and plays a role in the pathogenesis of septic shock or severe Gram positive induced inflammatory responses against bacterial infection. ${ }^{8}$

Pooja et al. ${ }^{9}$ have indicated that LTA plays an important role in host cell internalization and Cytotoxicity induction of Streptococcus agalactiae through energy-dependent Endocytic process. ${ }^{9}$ In the context of their research, Zheng et al. ${ }^{10}$ mimic septic condition in $\mathrm{H} 9 \mathrm{c} 2$ cells in the presence of lip polysaccharide (LPS) plus peptidoglycan (PG). Treatment by LPS plus PGN increased injury markers such as creatine kinase, lactate dehydrogenate, interleukin (IL)-6 and tumor necrosis factor (TNF)- $\alpha$ in the culture supernatant, reduced mitochondrial membrane potential, increased reactive oxygen species production and reduced ATP levels, thus indicating that bacteria affects mitochondria inducing mitochondrial dysfunction. ${ }^{10}$

Research employing LTA are related to prevention of damage with flavonoids. Flavonoids are secondary metabolites; they are a family of phenolic compounds practically ubiquitous in plants and in various fruits, leaves and seeds. ${ }^{11}$ Chemically, flavonoids are derivatives of benzo-c-pyrone and are composed of fifteen backbone carbon atoms sharing a common C6-C3-C6 skeleton. ${ }^{12}$ They are classified into six different groups, such as chalcones, flavones, flavonols, flavandiols, anthocyanins and proanthocyanins. Actually, flavonoids have commanded great interest due to their beneficial health effects; they present diverse biological properties such as: Anti-inflammatory, antioxidant, anticancer, antibacterial and antiviral agent. Thus the need arises to carry out new research in order to propose new alternative treatments.

Flavonoid apigenin $(2-15 \mu \mathrm{M})$ prevents, in a dose-dependent fashion, damage induced by LTA $(15 \mu \mathrm{g} / \mathrm{ml})$ including ІкB 
degradation, NF- $\mathrm{kB}$ translocation, MAPK activation, mRNA levels of COX-2 and IL-1 $\beta$ without cytotoxic effects in H9c2 cells, thus indicating that pre-treatment with apigenin could be used in inflammatory diseases and could be used as a therapeutic agent in IE. ${ }^{13}$ Another study has demonstrated pre-treatment with flavonoids kaempherol, apigenin, naringin and epigallocatechin gallate $(10 \mu \mathrm{M}$

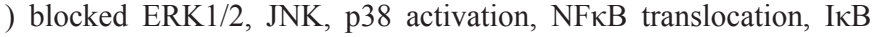
degradation, iNOS expression and NO production induced by LTA $(15 \mu \mathrm{g} / \mathrm{ml})$ obtained from Streptococcus sanguinis. All these effects regulated inflammatory response and additionally all flavonoids did not exhibit cytotoxic effects; therefore they could become suitable agents to prevent IE. ${ }^{7}$ Luteolin and quercetagetin $(10 \mu \mathrm{M})$ prevented ERK, p-38, JNK, AKT activation. Genistein and quercetin reduced its effects induced by ALT $(15 \mu \mathrm{M})$. All flavonoids reduced IL- $1 \beta$ expression and I $\mathrm{K} \mathrm{B} \alpha$ activation induced by treatment with ALT. The Flavonoids that were employed prevented inflammation and could be used in inflammatory diseases such as IE. ${ }^{14}$

\section{Concluding remarks}

Treatments with PGN and LTA induce mitochondrial dysfunction in $\mathrm{H} 9 \mathrm{c} 2$ cells. It is well known that mitochondria is the major source of reactive oxygen species (ROS), therefore, mitochondrial dysfunction could increase its production inducing catastrophic damage to the cells. However antioxidants as flavonoids could be employed to scavenge ROS and to reduce this damage. Moreover, flavonoids present antiinflammatory properties without cytotoxic effects in $\mathrm{H} 9 \mathrm{c} 2$ cells and do not generate resistance as opposed to antibiotic therapy. However, new research should target whether these compounds could provide the aforementioned benefits in humans and in a not too distant future manufacture oral products with flavonoids for the prevention of IE, cavities, plaque formation and others.

\section{Acknowledgements}

We wish to express our gratitude to DGAPA-UNAM for the postdoctoral fellowship and for-IN-201816 for providing funding necessary to the present.

\section{Conflict of interest}

The author declares no conflict of interest.

\section{References}

1. Hoen B, Duval X. Infective endocarditis. NEnglJMed.2013;368(15):14251433 .
2. Chen YY, Chen JC, Lin YC, et al. Endogeus molecules induced by a pathogen-associated molecular pattern (PAMP) elicit innate immunity in shrimp. PLoS One. 2014;9(12):1-21.

3. Trdá L, Boutrot F, Claverie J, et al. Perception of pathogenic or beneficial bacteria and their evasion of host immunity: pattern recognition receptors in the frontline. Front Plant Sci. 2015;6:219.

4. Errington J. Cell wall-deficient, L-form bacteria in the 21 st century: a personal perspective. Biochem Soc Trans. 2017;45(2) 287-295.

5. Hong YF, Lee Hy, Jung BJ, et al. Lipoteichoic acid isolated from Lactobacillus plantarum down-regulates UV-induced MMP-1 expression and up-regulates type I procollagen through the inhibition of reactive oxygen species generation. Mol Immul. 2015;67(2):248-255.

6. Gutiérrez-Venegas G, Cardoso-Jiménez P. Lipoteichoic acid promotes nuclear accumulation of beta-catenin via AKT in human gingival fibroblasts. Int Int Immunopharmacol. 2011;11(9):1278-1284.

7. Gutiérrez-Venegas G, Ventura-Arroyo JA, Arreguín-Cano JA, et al. Flavoids inhibit iS production via mitogen activated proteins in lipoteichoic acid stimulated cardiomyoblasts. Int Immunopharmacol. 2014;21(2):320-327.

8. Lee P Tan KS. Effects of Epigallocatechin gallate against Enterococcus faecalis biofilm and virulence. Arch Oral Biol. 2015;60(3):393-399.

9. Pooja S, Pushpanathan M, Gunasekaran P, et al. Endocytosismediated invasion and pathogenicity of Streptococcus agalactiae in rat cardiomyocyte (H9C2). PLoS One. 2015;10(10):1-16.

10. Zheng G, Lyu J, Liu S, et al. Silencing of uncoupling protein 2 by small interfering RNA aggravates mitochondrial dysfunction in cardiomyocytes under septic conditions. Int J Mol Med. 2015;35(6):1525-1536.

11. Tapas A, Sakarkar D, Kakde R. Flavoids as nutraceuticals: a review. Trop J Pharm Res. 2008;7(3):1089-1099.

12. Kumar S, Pandey AK. Chemistry and biological activities of flavoids: An overview. Scientific WorldJournal. 2013;29:162750..

13. Gutiérrez-Venegas G, González-Rosas Z. Apigenin reduce lipoteichoic acid-induced inflammatory response in rat cardiomyoblast cells. Arch Pharm Res. 2017;40(2):240-249.

14. Gutiérrez-Venegas G, Bando-Campos CG. The flavoids luteolin and quercetagetin inhibit lipoteichoic acid actions on H9c2 cardiomyocytes. Int Immunopharmacol. 2017;10(9):1003-1009. 